\title{
Kinderperspektiven auf institutionalisierte Elterngespräche in Kindertageseinrichtungen
}

\author{
Britta Menzel
}

Eingegangen: 1. April 2021 / Angenommen: 6. Juli 2021 / Online publiziert: 12. Januar 2022

(C) Der/die Autor(en) 2022

Zusammenfassung In Deutschland ist der institutionalisierte Austausch zwischen Eltern und Fachkräften in der pädagogischen Praxis als ,Elterngespräch ‘ mittlerweile fest verankert. Während in Elterngesprächen über Kinder gesprochen wird, ist bislang nicht bekannt, welche Perspektiven Kinder auf diese haben. Im vorliegenden Beitrag bilden Aussagen von Kindern zu Elterngesprächen den Analysegegenstand. Grundlage hierfür sind qualitative (Gruppen-)Interviews $(n=32)$ mit Kindern zwischen 3-6 Jahren, die 2017-2018 in einer Kindertageseinrichtung geführt wurden. Die Ergebnisse legen offen, was Kinder über Elterngespräche wissen und wie sie das Sprechen der erwachsenen Beteiligten über sie einschätzen. Auch zeigt sich, dass die Kinder unterschiedlich ausgeprägte Wünsche über Informiertheit und Involviertheit zu Elterngesprächen haben. Insgesamt lässt sich konstatieren, dass die Kinder ihre Perspektiven auf Elterngespräche in den Kontext generationaler Ordnung stellen.

Schlüsselwörter Elterngespräche · Kinderperspektiven · Qualitative Interviews · Frühkindliche Bildung 


\title{
Children's perspectives on formal parent-staff-conversations in early childhood settings
}

\begin{abstract}
In Germany, formal parent-staff-conversations are well established in pedagogical practice. In parent-staff-conversations adults mainly talk about children, however, little is known about how children perceive these conversations. Therefore, this paper examines children's statements on parent-staff-conversations. The data basis constitutes qualitative interviews $(n=32)$ with children between $3-6$ years, which were conducted between 2017-2018 in an ECEC setting. Results reveal what children know about parent-staff-conversations and how children review what is being said about them. Furthermore, one can see that children's wishes to be informed about the content of parent-staff-conversations and to what extent they might want to be included in these conversations can differ greatly. Finally, it can be concluded, that children's perspectives on parent-staff-conversations are interlinked with generational orders.
\end{abstract}

Keywords Parent-staff-conversations · Children's perspectives · Qualitative interviews $\cdot$ Early childhood education

\section{Einleitung}

Mit der zunehmenden Inanspruchnahme frühkindlicher Bildungs-, Betreuungs- und Erziehungsangebote ist auch das Verhältnis von Familie und Kindertageseinrichtung zum Gegenstand politischer, fachwissenschaftlicher und -praktischer Diskurse geworden (für einen Überblick: Betz et al. 2017). Im Vordergrund steht dabei die Forderung nach einem gemeinsamen, partnerschaftlichen Handeln von Eltern ${ }^{1}$ und Fachkräften, das dem Wohl des Kindes dienen und zugleich kompensatorisch auf vermeintliche Erziehungsdefizite wirken soll (Cloos und Karner 2010, S. $171 \mathrm{f}$.). Als ein zentrales Instrument der Zusammenarbeit wird unter anderem der Austausch zwischen Eltern und Fachkräften gesehen. So heißt es beispielsweise im Bayerischen Kinderbildungs- und -betreuungsgesetz (BayKiBiG) zur Erziehungspartnerschaft ${ }^{2}$ : „Die pädagogischen Fachkräfte informieren die Eltern regelmäßig über den Stand der Lern- und Entwicklungsprozesse ihres Kindes in der Tageseinrichtung“" (Teil 4, Art. 11). Dabei kann zwischen informellen Kontaktmomenten wie Tür- und Angelgesprächen und einem terminierten, bilateralen Austausch - sogenannten Elterngesprächen - unterschieden werden.

Elterngespräche als institutionalisierte Form des Austauschs richten sich an Erwachsene und nehmen vornehmlich Beobachtungen der Fachkräfte zum Handeln und Lernen der Kinder zum Ausgangspunkt (vgl. Kesselhut 2015). Die Anlage von Elterngesprächen sieht demnach vor, dass über Kinder gesprochen wird, während

\footnotetext{
1 Erziehungsberechtigte sind im Folgenden miteingeschlossen.

2 Der Begriff der Bildungs- und Erziehungspartnerschaft wird insbesondere in politischen und fachpraktischen Diskursen verwendet und impliziert programmatische Forderungen zur Zusammenarbeit von Eltern und Fachkräften (kritisch hierzu: z. B. Betz 2015).
} 
Kinder selbst nicht als Teilnehmende konzipiert $\operatorname{sind}^{3}$ (vgl. Betz et al. 2019a). In Elterngesprächen verschränken sich so verschiedene Lebensbereiche der Kinder miteinander. Dies gilt jedoch nicht nur für den Moment des Gesprächs, sondern auch für damit verbundene Konsequenzen, die sich für die Kinder aus dem wechselseitigen Zuspielen von Informationen der Eltern und Fachkräfte ergeben (können). Aus diesem Grund erstaunt es, dass Forschungen zu Elterngesprächen bislang vornehmlich die beteiligten Erwachsenen in den Blick genommen haben (u. a. Kesselhut 2015; Zehbe 2021). Welche Perspektiven Kinder auf Elterngespräche in frühpädagogischen Betreuungseinrichtungen haben, bildet somit eine Leerstelle in der sozialwissenschaftlichen Kindheitsforschung. Im vorliegenden Beitrag wird daher entlang inhaltsanalytischer Auswertungen (Kuckartz 2018) qualitativer (Gruppen-)Interviews mit Kindern zwischen drei bis sechs Jahren nachgezeichnet, welche Aussagen Kinder über Elterngespräche treffen. Daraus lässt sich einerseits ersehen, was Kinder selbst über Elterngespräche wissen und andererseits, wie sie über eine (mögliche) Teilnahme an Elterngesprächen denken.

Im Folgenden wird zunächst ein Überblick zu Studien gegeben, die Elterngespräche empirisch untersuchen und dabei auch die Perspektiven von Kindern zum Gegenstand haben. Im Anschluss daran wird die theoretische Verortung sowie das methodische Vorgehen vorgestellt. Abschließend erfolgt Darstellung und Diskussion der Analysenergebnisse.

\section{Forschung zu Kinderperspektiven auf institutionalisierte Elterngespräche}

Institutionalisierte Elterngespräche in Kindertageseinrichtungen werden bislang vornehmlich entlang von drei Schwerpunkten untersucht. Erstens werden Gesprächsverläufe zwischen Eltern und Fachkräften in den Blick genommen und im Zusammenhang diskursiver Aushandlungsprozesse und asymmetrischer Machtverhältnisse analysiert (u. a. Betz et al. 2021; Cloos et al. 2013; Karila 2006; Kesselhut 2015; Zehbe 2021). Zweitens werden Gespräche von Eltern und Fachkräften in Studien zur Bildungsdokumentation im frühkindlichen Bildungs-, Betreuungs- und Erziehungsbereich zum Forschungsgegenstand (z. B. Kuhn et al. 2018; Urban et al. 2015). Drittens untersuchen Studien, inwiefern Kinderperspektiven in Elterngesprächen von den erwachsenen Teilnehmenden berücksichtigt werden (Alasuutari 2014) und wie Eltern und Fachkräfte über eine (mögliche) Teilnahme von Kindern an diesen Gesprächen denken (Betz et al. 2019a). Studien, die sich explizit mit den Perspektiven von Kindern auf Elterngespräche auseinandersetzten, lassen sich bisher nur für den Schulbereich finden. Elterngespräche im Schulbereich können an verschiedenen Punkten von denen in Kindertageseinrichtung abgegrenzt werden, etwa indem Schülerinnen und Schüler auch als (Gesprächs-)Teilnehmende konzipiert

\footnotetext{
${ }^{3}$ Dies bezieht sich auf das ,klassische` Verständnis von Elterngesprächen. Durchaus lassen sich trägerspezifisch oder in einzelnen Einrichtungen Formen der Zusammenarbeit finden, in denen auch Kinder in Gespräche involviert sind. Inwieweit diese dann Elterngespräche ersetzen oder als solche bezeichnet werden, kann jedoch nicht generalisiert werden.
} 
werden (vgl. Bonanati 2018, S. 13f.). Die empirischen Erkenntnisse lassen sich daher nicht ohne weiteres übertragen. Dennoch bieten sich interessante Anknüpfungspunkte für die vorliegende Fragestellung, weshalb im Folgenden auf einige näher eingegangen wird.

In einer quantitativ angelegten Studie von Croizer (1999) wurden mittels Befragungsbögen die Sichtweisen von Eltern, Lehrkräften und Kindern auf die Zusammenarbeit in drei weiterführenden Schulen erhoben. Croizer kann zeigen, dass die Mehrheit der Schülerinnen und Schüler eine positive Einstellung zu den Gesprächen zwischen Eltern und Lehrkräften hat. Für die Teilnahme der Schülerinnen und Schüler an Elterngesprächen lassen sich die Ergebnisse u.a. mittels sozialer Merkmale (Alter, Geschlecht) weiter ausdifferenzieren. So möchten beispielsweise einige in Elterngespräche involviert sein, wohingegen andere aus Sorge vor dem, was besprochen wird, nicht teilnehmen möchten. In der Studie von Ericsson und Larsen (2002) wird bei der Analyse von Beobachtungen in Elterngesprächen und qualitativer Interviews mit Schülerinnen und Schüler im Alter von 7 und 13 Jahren konstatiert, dass Kinder den Austausch zwischen Eltern und Lehrkräften mitunter kritisch bewerten, etwa indem sie negative Konsequenzen für sich befürchten. Gleichzeitig wird insbesondere in den Beobachtungen sichtbar, dass Kinder Elterngespräche auch nutzen, um eigene Anliegen (mit Hilfe ihrer Eltern) vorzutragen. Eine weitere Studie, die die Perspektiven von Grundschülerinnen und -schüler auf das Verhältnis zwischen Schule und Familie in den Blick nimmt, ist die Untersuchung von Betz et al. (2019b). Mittels Gruppendiskussionen rekonstruieren die Autorinnen unter Bezugnahmen auf Elterngespräche unterschiedliche Typen kollektiver Handlungsorientierungen der Schülerinnen und Schüler. Darin wird sichtbar, dass sich die Schülerinnen und Schüler, vergleichbar mit den Ergebnissen von Ericsson und Larsen (2002), sowohl als Profiteur/innen des Austauschs zwischen Eltern und Lehrkräften als auch als Ausgeschlossene erleben können. Auch Stiller (2020) fragt in seiner qualitativen Studie zur Kindersicht auf ,Elternarbeit' in Grundschulen danach, was Schülerinnen und Schüler zur Zusammenarbeit von Eltern und Lehrkräften sagen. Analysegrundlage bilden Gruppendiskussionen, in denen zum einen sichtbar gemacht wird, dass Kinder den formellen Austausch von Eltern und Lehrkräften zu Elternsprechtagen als ,emotionale Belastung erleben“ (ebd., S. 302). Zum anderen zeigt sich, dass Kinder mehrheitlich die eigene Teilnahme an diesen Gesprächen als langwierig beschreiben.

Insgesamt wird in den Schulstudien zweierlei sichtbar. Zum einen zeigt sich, dass es keine einheitliche Perspektive auf Elterngespräche gibt, sondern Kinder unterschiedliche Sichtweisen zum Austausch von Eltern und Lehrkräften einnehmen. Zum anderen wird deutlich, dass die (Nicht-)Teilnahme von Kindern an Elterngesprächen ein wichtiger Einflussfaktor dafür ist, wie sich Schülerinnen und Schüler zu dem Austausch von Eltern und Lehrkräften äußern. Inwieweit sich für den frühkindlichen Bildungs-, Betreuungs- und Erziehungsbereich vergleichbare Einschätzungen von Kindern finden lassen, ist Teil des Erkenntnisinteresses dieses Beitrags. 


\section{Theoretische Rahmung: generationale Ordnung als kindheitstheoretisches Konzept}

Die aufgeführten Studien aus dem Schulbereich deuten darauf hin, dass Gespräche zwischen Kindern, Eltern und Lehrkräften von asymmetrischen, generationalen Machtverhältnissen unterlegt sind und sich dies in den Perspektiven von Kindern auf Elterngespräche spiegelt. Daher wird im Folgenden auf das Konzept der generationalen Ordnung zurückgegriffen, um die Anlage von Elterngesprächen theoretisch nachvollziehbar zu machen.

Mit dem Konzept der generationalen Ordnung werden ,Kind “ und ,Erwachsene/r als sozial hervorgebrachte Kategorien begriffen, die sich erst in Abhängigkeit zueinander zeigen (Alanen 2011). Die Kategorien ,Kind“ und ,Erwachsene/r' können als gesellschaftliche Strukturkategorien verstanden werden, denen vornehmlich das Alter als Differenzlinie zugrunde liegt. In der sozialwissenschaftlichen Kindheitsforschung sind diese Kategorien eng mit generationalen Ungleichheiten verbunden. Mit diesem Konzept werden vornehmlich asymmetrische Machtgefüge zwischen Kindern und Erwachsenen in den Blick gerückt, etwa indem Kindern abgesprochen wird, gewisse Kompetenzen mitzubringen oder eine eigene Meinung zu haben (Bühler-Niederberger 2014, S. 341). Dementsprechend geht es dabei nicht um Altersordnungen, sondern um „Ordnungen der Macht und der Ungleichheit“ (Honig 2018, S. 199). Gleichzeitig wird davon ausgegangen, dass Kinder im Sinne einer Komplizenschaft an der Reproduktion entsprechender Machtverhältnisse beteiligt sind, indem ,sie eine generationale Ordnung nicht nur akzeptieren“ sondern ,in deren beständiger Herstellung kooperieren“ (Bühler-Niederberger 2020, S. 238).

Neben der bisherigen Eingrenzung der generationalen Ordnung auf die binäre Unterscheidung zwischen Kindern und Erwachsenen plädieren einige Kindheitsforschende dafür, das Konzept differenzierter zu betrachten. So fordert Kelle (2018), sich mehr mit der ,lebensalterspezifischen Variabilität der Abhängigkeit und damit auch der agency von Kindern“ (ebd., S. 48, Hervorh. im Orig.) zu beschäftigen, d. h. im Forschungskontext auch das Alter der beteiligten Kinder in Bezug auf generationale Ordnung zu berücksichtigen. Demnach gilt es in den Blick zu nehmen, dass sich die Angewiesenheit von Kindern auf Erwachsene mit zunehmendem Alter verändert und eine binäre Unterscheidung zwischen Kindern und Erwachsenen daher zu kurz greift. Auch Honig (2018) schlägt eine entsprechende Erweiterung vor und kritisiert, dass das von Alanen (2011) vertretene binär ausgerichtete Konzept der generationalen Ordnung insbesondere für das Verhältnis zwischen Eltern und Kindern zu kurz greift. Damit wird ,die Ambiguität von Autonomie und Verbundenheit in den Beziehungen von Kindern und Eltern“ (Honig 2018, S. 200) verkannt.

Für die empirische Auseinandersetzung mit Elterngesprächen werden entsprechende Perspektiven anschlussfähig, die generationale Ordnung multidimensional verstehen, denn auch hier sind verschiedene Generationenbeziehungen verbunden: Kinder, Eltern und Fachkräfte. Elterngespräche verweisen bereits qua Bezeichnung auf generationale Prämissen: Setzt von Kindern zu sprechen, eine Vorstellung von Erwachsenen voraus (Honig 2018, S. 194), können Elterngespräche auch nur Bedeutung erhalten, wenn Kinder den Ausgangspunkt bilden. So wird die triadische Verwobenheit der generationalen Kategorien Kinder, Eltern, Fachkräfte auch zur Vo- 
raussetzung, Elterngespräche als solche bezeichnen zu können. Aus diesem Grund ist es Ziel darzustellen, inwieweit Bedingungen generationaler Ordnung den Aussagen von Kindern zugrunde liegen.

\section{Methode und Sampling}

Ausgangspunkt der vorliegenden Analyse bildet ein Promotionsprojekt an der Johannes Gutenberg-Universität Mainz, in dem mittels ethnographischer Beobachtungen und qualitativer Interviews die Akteurschaft von Kindern im Verhältnis von Familie und Kindertageseinrichtung explorativ untersucht wird (hierzu auch Menzel 2021), wobei in diesem Beitrag die Interviews als Datengrundlage dienen.

Qualitative Interviews sind ein bewährtes Erhebungsinstrument in der sozialwissenschaftlichen Kindheitsforschung, um „Befunde über Lebens- und Erfahrungswelten von Kindern [zu] generieren“ (Andresen 2012, S. 138). Grundlegende Annahme ist, dass Kinder ,bereits über vielfältige Kompetenzen und komplexes (Erfahrungs-) Wissen verfügen" (Nentwig-Gesemann und Mackowiak 2012, S. 123), so dass mittels qualitativer Interviews „Prozesse des Verstehens und Erklärens von Kindheit, Kindern und generationalen Ordnungen“ (ebd.) möglich werden. Um zu generieren, welche Aussagen Kinder zu Elterngesprächen treffen, wurden Einzel- und Gruppeninterviews $(n=32)$ mit 18 Kindern zwischen drei bis sechs Jahren inhaltsanalytisch ausgewertet (Kuckartz 2018). Die Erhebungen fanden 2017-2018 im Rahmen regelmäßiger wöchentlicher Aufenthalte in einer Kindertageseinrichtung in freier Trägerschaft statt, die sowohl Krippen- als auch Kindergartengruppen führt. Die Einrichtung liegt im Randgebiet eines privilegierten Stadtviertels einer bayerischen Großstadt. In dieser Einrichtung ist ein jährlich stattfindender Austausch zwischen Eltern und Fachkräften zum Entwicklungsstand des Kindes vorgesehen und wird von den Erwachsenen und Kindern selbst als „Elterngespräch“ bezeichnet.

Die Perspektiven von Kindern als Erkenntnisinteresse empirischer Erhebungen werden in der Kindheitsforschung kritisch diskutiert (u. a. Heinzel et al. 2012; Honig 1999; Mey 2013). So wird mehrheitlich konstatiert, dass Forschende nur aus ihrer Erwachsenenperspektive auf die verbalen Erzeugnisse von Kindern schauen können und entsprechend „Erfahrungen und Interessen von Kindern immer nur stellvertretend artikulieren“ (Heinzel 2012a, S. 180). Unter dieser Prämisse ist die systematische Analyse von Aussagen der Kinder zu Elterngesprächen als Annäherung an die unterschiedlichen Perspektiven von Kindern auf den institutionalisierten Austausch von Eltern und Fachkräften zu verstehen. Ausgehend davon wird das Vorgehen im Folgenden ausführlich beschrieben, um auch generationale Bedingungen im Erhebungsverfahren nachvollziehbar zu machen.

Für die Datenerhebung wurden im Vorfeld ethische Richtlinien festgelegt und im Forschungsprozess stetig reflektiert (siehe z.B. Cameron 2005) ${ }^{4}$. Die Kinder durften beispielsweise selbst entscheiden, ob sie alleine oder mit von ihnen bestimmten Kin-

\footnotetext{
4 Ausführlich wird das Erhebungsverfahren sowie damit verbundene forschungsethische Herausforderungen und Reflexionen in Folgepublikationen diskutiert. Aufgrund des Schwerpunkts auf den empirischen Analysen wird es hier nur auszugsweise dargestellt.
} 
dern an den Interviews partizipieren ${ }^{5}$ (vgl. Brenneke und Tervooren 2019, S. 205). Damit war es den Kindern möglich, ein Gegengewicht zur „generationenbedingten Dominanz" (Heinzel 2012b, S. 104) in Interviewsituationen herzustellen. Gleichzeitig kann die Teilnahme mehrerer Kinder dazu beitragen, dass durch das Gespräch unter Freundinnen und Freunden ,dem erwachsenen Zuhörer erweiterte Möglichkeiten“ (Weltzien 2012, S. 144) geboten werden, ihre Perspektiven zu verstehen. Im Erhebungsverlauf kamen die Kinder auch selbstläufig auf die Forschende zu, um „wieder das mit dem Mikrofon“ ( $\operatorname{Sarah}^{6}, 6$ Jahre) machen zu dürfen. Entsprechend wurde das Sample dadurch bestimmt, wie die Kinder die Interviewgruppen selbst zusammensetzten. So nahmen insgesamt zehn Mädchen und acht Jungen an den Interviews teil.

$\mathrm{Zu}$ Beginn der Interviews wurde das Forschungsvorhaben vorgestellt, auf die Vertraulichkeit des Gesprächs verwiesen und die Einwilligung zur Teilnahme erfragt. Auch wurde über den Ablauf informiert (z.B. freies Erzählen, Möglichkeit des Abbruchs) (vgl. Cameron 2005, S. 599). Die Interviews wurden dann mit einem offenen Einstiegsstimulus eingeleitet (z. B. „Magst du erzählen, was so passiert, wenn du morgens im Kindergarten ankommst?"). Damit konnten eigenständige Erzählungen der Kinder angeregt werden (vgl. Brooker 2007, S. 167). Im weiteren Verlauf wurden entlang eines Leitfadens verschiedene, für das Erkenntnisinteresse relevante Themen von der Forscherin aufgegriffen (u. a. Elterngespräche), sofern diese nicht selbstläufig von den Kindern angesprochen wurden. Dabei wurden die verschiedenen Themen ebenfalls offen eingeleitet. So wurde beispielsweise zunächst allgemeiner nach dem Austausch von Eltern und Fachkräften gefragt (z. B. „Unterhalten sich eure Eltern auch mal mit der Miriam [Fachkraft] und wie ist das so? Was passiert da?"). Ziel dabei war es zu eruieren, welche eigenen Relevanzsetzungen die Kinder vornehmen (u. a. Tür- und Angelgespräche, Hausbesuche, Elterngespräche). Im Weiteren wurden dann themenfokussierendere Nachfragen gestellt, um die Kinder auch zu spezifischen Themen sprechen zu lassen (z. B. „Und kennt ihr Elterngespräche?"). Dennoch wurden auch Grenzen in der Erhebung durch qualitative Interviews sichtbar. Kindern fällt es leichter, „,ich zu aktuellen Erfahrungen und Ergebnissen zu äußern als zu weit zurückliegenden Ereignissen oder Zukunftsperspektiven" (Nentwig-Gesemann und Mackowiak 2012, S. 121). Dementsprechend waren die Möglichkeiten der Kinder, sich zu Elterngesprächen zu äußern, potenziell dadurch beeinflusst, wie weit ein Elterngespräch zurücklag oder inwiefern sie auch selbst involviert waren.

Für die vorliegende Analyse wurden entlang der inhaltlich strukturierenden Inhaltsanalyse (Kuckartz 2018, S. $97 \mathrm{ff.}$.) all jene Sequenzen berücksichtigt, die der thematischen Hauptkategorie ,Elterngespräche' in einem ersten Schritt zugeordnet

\footnotetext{
5 Dementsprechend beinhaltet das vorliegende Material sowohl Einzel- als auch Gruppeninterviews, wobei mehr als zwei Drittel Gruppeninterviews mit 2-3 Kindern sind. Wenngleich aus methodisch-methodologischer Sicht durchaus berechtigte Differenzierungen zwischen Einzelinterviews, Gruppeninterviews und Gruppendiskussionen in Bezug auf das jeweilige Erkenntnisinteresse gezogen werden (Nentwig-Gesemann 2002, S. 45), wurde im eigenen Vorgehen den Präferenzen der Kinder, in welchem Rahmen sie die Gespräche mit der Forscherin führen möchten, Vorrang geboten. Im Anschluss an Lange und Mierendorff werden daher Gruppendiskussionen als ,Variante der qualitativen Interviews“ (2009, S. 200) verstanden.

${ }^{6}$ Im vorliegenden Beitrag wurden alle Personennamen pseudonymisiert.
} 
wurden. Ausgewertet wurden folglich die Aussagen der Kinder, in denen sie auf Elterngespräche Bezug nehmen. Dabei handelt es sich einerseits um Sequenzen, in denen Kinder selbstläufig über Elterngespräche sprechen. Andererseits wurden ebenfalls jene Sequenzen berücksichtigt, in denen Kinder auf die Fragen der Forscherin zu Elterngesprächen antworten. Für die weitere Kategorienbildung wurde daher im zweiten Schritt ein induktives Vorgehen gewählt, indem zunächst einzelne Sequenzen paraphrasiert und dann zu ersten Subkategorien gefasst wurden. Im dritten Schritt konnten diese Subkategorien in drei übergeordnete Kategorien gruppiert werden, um so das Material weiter zu systematisieren: (1) Wissen über Elterngesprächen, (2) Einschätzungen zu Elterngesprächen und die (3) Teilnahme von Kindern an Elterngesprächen. Dabei wurde sichtbar, dass die Aussagen von Kindern sowohl Wissen über als auch Einschätzungen zu Elterngesprächen beinhalten. Auch eine (mögliche) Teilnahme wurde sowohl selbstläufig als auch auf Nachfrage der Forscherin thematisiert. Entlang des hierarchischen Kategoriensystems wurde in einem vierten Schritt das gesamte Material erneut codiert. Zuletzt erfolgte eine kategorienbasierte Auswertung ${ }^{7}$, die für die anschließende Ergebnisdarstellung die Grundlage bildet.

\section{Elterngespräche als exklusiver Informationsaustausch zwischen Erwachsenen - Empirische Einblicke}

Die drei zuvor genannten übergeordneten Kategorien umfassen die wesentlichen Aspekte, die von den Kindern im Bezug auf Elterngespräche geäußert wurden. Hierzu werden im Folgenden die Analyseergebnisse entlang der darin zugeordneten Subkategorien vorgestellt.

\subsection{Wissen über Elterngespräche}

In den Interviews zeigt sich, dass die befragten Kinder vielfältige Vorstellungen davon haben, was Elterngespräche sind. Das Wissen von Kindern über Elterngespräche ergibt systematisiert vier Subkategorien: Strukturmerkmale von Elterngesprächen (u.a. Wo finden Elterngespräche statt?, Wer nimmt teil?), Begründungen, warum Elterngespräche notwendig sind, und Inhalte von Elterngesprächen. Darüber hinaus wurden hier auch Sequenzen zugeordnet, in denen Kinder aussagen, über Elterngespräche nichts zu wissen.

Hinsichtlich der Strukturmerkmale von Elterngesprächen zeigt sich, dass die Kinder den institutionalisierten Austausch zwischen Eltern und Fachkräften vornehmlich als Orte der Exklusivität beschreiben (z. B. „da dürfen nur die Eltern hin“ (Verena, 6 Jahre)). Auch in den Begründungen, warum Elterngespräche stattfinden, wird

\footnotetext{
7 Für eine kategorienbasierte Auswertung werden nach Kuckartz (2018) die Hauptkategorien herangezogen. Aufgrund des spezifischen Erkenntnisinteresses im vorliegenden Beitrag zu Kinderperspektiven auf Elterngespräche wird lediglich die Hauptkategorie ,Elterngespräche“ zugrunde gelegt und dementsprechend die daraus hervorgegangenen Subkategorien als Ausgangspunkt der kategorienbasierten Auswertung herangezogen.
} 
deutlich, dass das Wissen über Elterngespräche von generationalen Ordnungsvorstellungen unterlegt ist, wie folgende Aussage illustriert: „die [Eltern, Anm. d. V.] hören ja alles nicht. Paar Kinder wollen es ja nicht sagen, wenn die fragen ,Und wie war's heute im Kindergarten“ und deswegen“" (Anabell, 5 Jahre). Die Legitimität des Informationsaustauschs von Erwachsenen über Kinder wird hier nicht in Frage gestellt.

Die Inhalte von Elterngesprächen beziehen sich den Kindern zufolge auf den Kindergarten. Dies wird in verschiedenen Sequenzen sichtbar. So beschreibt ein Kind beispielsweise, dass Erwachsene ,über nur Kindergarten BlaBlaBla“ (Xaver, 5 Jahre) sprechen würden. Während manche Aussagen vage bleiben und sich in dem „BlaBlaBla“ auch eine gewisse Bedeutungslosigkeit der Gesprächsinhalte für Kinder ausdrückt, spezifizieren andere Kinder die Gesprächsinhalte. Demzufolge geht es in Elterngesprächen um die Rückmeldungen von Fachkräften an Eltern zum Geschehen in der Kindertageseinrichtung: „Dann sagen die Erzieherinnen, wie es läuft im Kindergarten“ (Verena, 6 Jahre). Aus den hier zugeordneten Sequenzen geht hervor, dass es dabei insbesondere um das (,schlechte') Verhalten der Kinder geht. So würden Eltern in den Gesprächen von Fachkräften darüber informiert, wenn sich ein Kind aus ihrer Sicht falsch verhalten hat. Ein Kind beschreibt dies wie folgt: „Wenn meine Mama nach Hause kommt und und mir sagt zum Beispiel ,Anabell, hast du heute die Monica gezwickt oder gebissen?"“ (Anabell, 5 Jahre). Auch die Lernentwicklung der Kinder wird in einigen Sequenzen als Gegenstand von Elterngesprächen beschrieben, wobei dies ausschließlich im Zusammenhang zu bevorstehenden Einschulungen aufgegriffen wurde: „Die haben gesagt, sie haben über gesprochen, äh, die Schule, ähm, ist der gut im Kindergarten, ist er gut in der Vorschule, macht der alles gut" (Tim, 6 Jahre).

Neben diesen inhaltlichen Beschreibungen von Elterngesprächen gab es auch Aussagen, in denen Kinder berichteten, nicht zu wissen, worüber Eltern und Fachkräfte sprechen. So antworten einige Kinder auf die Frage zu den Themen von Elterngesprächen mit ,weiß ich nicht“ (Lara, 5 Jahre). Hierfür werden selbstläufig verschiedene Gründe benannt. Einerseits, dass die eigene Abwesenheit vom Elterngespräch dazu führt, keine Kenntnisse zum besprochenen Inhalt zu haben. Andererseits würden die Kinder von ihren Eltern nicht über die Inhalte informiert. Demzufolge steht die Zugänglichkeit von Wissen über Elterngespräche deutlich in Abhängigkeit dazu, inwieweit sie von den erwachsenen Teilnehmenden involviert und informiert werden. Auch hier wird die Exklusivität von Elterngesprächen entlang generationaler Differenzlinien sichtbar. Dabei gehen gleichzeitig die Meinungen der Kinder dazu auseinander, inwiefern sie wissen möchten, was Eltern und Fachkräfte besprechen. Während ein Kind beispielsweise den Wunsch äußert, mehr einbezogen zu werden, beschreiben andere Kinder es als positiv, nichts über die Inhalte von Elterngesprächen zu wissen, da sie es als ,langweilig“ (Luca, 5 Jahre) einschätzen.

Es wird deutlich, dass Kinder über vielfältiges Wissen zu Elterngesprächen verfügen, obwohl sie nur selten dabei sind. Dieses Wissen reicht von Strukturmerkmalen bis hin zu Gesprächsinhalten. Insbesondere die Bewertung von Verhalten und Leistung werden von den Kindern als zentrale Themen benannt. Kinder konzipieren ihr Verständnis von Elterngesprächen dabei entlang generationaler Ordnungsvorstellungen. Elterngespräche richten sich den Kindern zufolge an Eltern, womit die Kinder 
in dieser Studie auf das gängige Verständnis von Elterngesprächen rekurrieren. Daraus resultiert für sie auch die ,logische“ Konsequenz, selbst nicht in das Sprechen über sie beteiligt zu werden.

\subsection{Einschätzungen zu Elterngesprächen}

Die Aussagen von Kindern zu Elterngesprächen aus der vorliegenden Studie geben auch Aufschluss darüber, wie sie den Austausch zwischen Eltern und Fachkräften einschätzen. Zwei unterschiedliche Einschätzungen werden dabei sichtbar: Elterngespräche als Eingriff in die informationelle Selbstbestimmung und Elterngespräche als geduldeter Austausch über Erwachsenenbelange.

Elterngespräche werden besonders dann als Eingriff in die informationelle Selbstbestimmung beschrieben, wenn die Kinder davon berichten, dass ihr vermeintlich falsches Verhalten thematisiert wird. So beschreibt ein Kind, dass sie es „,blöd“ (Anabell, 5 Jahre) findet, wenn ihre Mutter im Elterngespräch von Streitereien im Kindergarten erfährt und sie im Nachgang darauf anspricht. Dabei wird deutlich, dass es Informationen gibt, von denen Kinder nicht wollen, dass sie weitergegeben werden. Eine Möglichkeit, sich der Weitergabe entgegenzustellen, ist aus ihrer Sicht nicht gegeben. Vielmehr scheint die Entscheidungsmacht über den Informationsaustausch ausschließlich bei den Erwachsenen zu liegen, worin sich generationale Ungleichheitsverhältnisse manifestieren. In einer anderen Sequenz wird der Austausch zwischen Eltern und Fachkräften als „unhöflich“ (Felix, 6 Jahre) beschrieben und der Wunsch geäußert, ,,dass die, dass wir gar nicht darüber reden“ (Tim, 6 Jahre). Es kann somit konstatiert werden, dass Kinder den Austausch über sich durchaus kritisch betrachten und sie Elterngespräche mitunter mit negativen Konsequenzen für sich assoziieren.

Im Gegensatz dazu zeigte sich auch, dass Kinder Elterngespräche dulden und dem Austausch über Erwachsenenbelange durchaus etwas abgewinnen, wenngleich es im Material der vorliegenden Studie hierzu nur eine Sequenz gibt, die dies explizit macht. So beschreibt ein Junge, unter welchen Bedingungen er Elterngespräche befürwortet: ,Aber manchmal, manchmal mag ich's, weil manchmal durfte ich allein in der, in meinem Schulraum alles angucken“ (Tim, 6 Jahre). Diese Einschätzung zu Elterngesprächen ist demzufolge weniger an den tatsächlichen Austausch von Eltern und Fachkräften geknüpft als vielmehr an damit verbundene Aktivitäten, die als besonders wahrgenommen werden.

\subsection{Teilnahme von Kindern an Elterngesprächen}

Die Teilnahme von Kindern an Elterngesprächen ist bislang in frühkindlichen Betreuungseinrichtungen nicht üblich (vgl. Betz et al. 2019a). Auch im vorliegenden Sample werden Kinder in der Regel nicht involviert, wie eine Sequenz verdeutlicht: „Aber ich glaub da sind immer keine Kinder dabei, weil das ja ein Elterngespräch ist" (Anabell, 5 Jahre). Dennoch finden sich im Material Sequenzen, in denen die Teilnahme von Kindern angesprochen wird. Dies bezieht sich erstens auf die eigene Erfahrung, an einem Elterngespräch teilgenommen zu haben. Zweitens beschreiben Kinder begrenzte Möglichkeiten teilzunehmen und drittens unter welchen Bedin- 
gungen sie selbst im Austausch von Eltern und Fachkräften (nicht) beteiligt werden möchten.

Kinder berichten davon, an Elterngesprächen teilgenommen zu haben, wenn es sich um ein Vorbereitungsgespräch zur Einschulung handelte oder wenn die Eltern anderweitig keine Betreuungsmöglichkeit für die Kinder hatten: „Wenn, wenn beide zum Elterngespräch gehen, müssen wir ja mit. Und, und immer gehen beide und deswegen muss ich dann mit" (Lara, 5 Jahre). Die Notwendigkeit einer Teilnahme wird jedoch aufgelöst, wenn ein „Kindermädchen“ (Verena, 6 Jahre) auf sie aufpasst.

In den Elterngesprächen nehmen die Kinder vornehmlich eine beisitzende und unbeteiligte Position ein, wie aus einigen Sequenzen hervorgeht. Ein Beispiel hierfür ist der folgende Interviewauszug, in dem sich Felix und Tim (6 Jahre) über ihre Erfahrungen austauschen.

Interviewerin: Und was hast du dann gemacht?

Felix: Nichts.

Interviewerin: Nichts?

Tim: Du konntest doch malen.

Felix: Nein.

Tim: Auch nicht?

Felix: Nein.

Die Kinder beschreiben an verschiedenen Stellen, dass sie sich verbal nicht ins Gespräch eingebracht haben. Unter anderem wird dies selbstläufig damit begründet, dass sie sich aus ihrer Position als Kind heraus nicht als aktiv Teilnehmende verstehen: „Ne, wir dürfen nicht, weil des ja ein Elterngespräch. Da, da kommt doch Eltern vor, dass nur Eltern sprechen dürfen" (Lara, 5 Jahre). Dies lässt den Schluss zu, dass Kinder die der Konzipierung von Elterngesprächen zugrundeliegende generationale Ausrichtung durch ihr Handeln anerkennen und situativ die damit verbundenen asymmetrische Machtverhältnisse komplizenhaft unterstützen. Dementsprechend wird die Möglichkeit einer eigenen verbalen Beteiligung nicht in Erwägung gezogen. Das stille Beisitzen erleben die Kinder als ,bumsbisel langweilig“ (Paul, 5 Jahre). Dieser Zustand kann dann verbessert werden, ,wenn man ein Kuscheltier oder ein Spielzeug dabei hat“ (Lara, 5 Jahre), denn dann sei es ,nicht so langweilig“" (ebd.).

Im Gegensatz zu den Erfahrungen der Teilnahme einzelner Kinder berichten andere, dass ihnen keine Möglichkeit gegeben wird, den Elterngesprächen beizuwohnen: „Also hat uns noch nie jemand gefragt“ (Sarah, 6 Jahre). Nicht teilnehmen zu dürfen, wird von einem Kind besonders kritisch gesehen: „Ich bin aber nie dabei, deswegen ist meine Mama gemein“ (Anabell, 5 Jahre). Dementsprechend berichten die Kinder in einigen Sequenzen, dass sie gerne an Elterngesprächen teilnehmen möchten, beispielsweise ,weil ich möchte hören, ob die was über mich reden“ (Anabell, 5 Jahre). Auch wenn Freundinnen oder Freunde dabei wären, können sich manche Kinder vorstellen, bei Elterngesprächen dabei zu sein, um gemeinsam ,im Flur [zu] spielen“ (Luca, 5 Jahre).

Die Inhalte des Austauschs von Eltern und Fachkräften werden auch als Faktor dafür benannt, inwieweit Kinder am Gespräch (nicht) beteiligt werden möchten. 
Dabei wird in einer Sequenz deutlich, dass sich Kinder auch hier eine zuhörende Position zuschreiben, wie es Sarah (6 Jahre) formuliert:

Interviewerin: Und würdet ihr gerne mehr dabei sein?

Sarah: Hm, wenn's keine wichtigen Gespräche sind nicht, aber wenn's was

Tolles ist zum Zuhören dann schon.

Mathilda: Ja.

Interviewerin: Ja? Was wär' denn was Tolles?

Sarah: Zum Beispiel, wann wir wieder Spielzeugtag machen. Oder offenes Haus.

Elterngespräche werden von den Kindern folglich als (potenzielle) Informationsquelle für Themen wahrgenommen, die für sie Bedeutung haben. Gleichzeitig wird deutlich, dass die Teilnahme durch Erwachsene reguliert wird. In den Interviews gibt es jedoch auch Sequenzen, in denen Kinder bekunden, kein Interesse daran zu haben, dabei zu sein. Dies wird primär damit begründet, dass der Austausch zwischen Eltern und Fachkräften als eintönig erlebt wird: „Früher war ich gern dabei. Aber jetzt nicht, weil es langweilig ist“" (Verena, 6 Jahre).

Insgesamt zeigt sich, dass das Interesse der Kinder, Elterngesprächen beizuwohnen, unterschiedlich ausgeprägt ist. Der Wunsch nach einer Teilnahme wird in Abhängigkeit zu unterschiedlichen Voraussetzungen und Rahmenbedingungen gestellt (Spielen, Freundinnen und Freunde, Themen). Kinder positionieren sich dabei primär als unbeteiligt. Deutlich wird, dass insbesondere jene Kinder, die bereits an Elterngesprächen teilgenommen haben, den Austausch von Eltern und Fachkräften durch generational asymmetrische Beteiligungsmöglichkeiten geprägt beschreiben.

\section{Schlussfolgerungen}

Die Ergebnisse verdeutlichen, dass die Perspektiven der Kinder zu Elterngesprächen mit deren konzeptioneller Anlage als ein exklusiver Austausch zwischen Erwachsenen übereinstimmen. Die Kinder beschreiben sich dabei primär als (zuhörende) Unbeteiligte und Erwachsene als Gesprächsteilnehmende. Sie begründen dies mit ihrer Position als Kind. Darin wird sichtbar, dass die Kinder sehr genau darum wissen, was generationale Ordnung ist und wie diese hergestellt wird. Auch wird eine hohe Übereinstimmung in den Sequenzen dahingehend deutlich, dass über Kinder gesprochen wird. Zentrale Gesprächsthemen in Elterngesprächen sind vornehmlich die Bewertung des Verhaltens und die Lernentwicklung von Kindern. Sie werden damit zum Gesprächsgegenstand von Eltern und Fachkräften. Die Kinder beschreiben Elterngespräche als gegen sie gerichtet, wenn es um das Recht auf informationelle Selbstbestimmung geht. Dabei gehen sie davon aus, nicht in den Austausch zwischen Eltern und Fachkräften eingreifen oder etwas an ihrer Position verändern zu können. Gleichzeitig ist jedoch festzuhalten, dass es von Kindern durchaus geduldet werden kann, wenn sich Erwachsene in Elterngesprächen austauschen.

Des Weiteren zeigen die Ergebnisse, dass Elterngespräche nicht nur als Austausch zwischen Erwachsenen markiert werden. Vielmehr lassen sich aus den Aussagen der Kinder weit differenziertere Schlüsse ziehen. So zeichnen die Kinder (implizit) die 
Fachkräfte als aktive Sprechende im Elterngespräch aus, indem sie ihre Befürchtungen zum Austausch der Erwachsenen darauf richten, dass Fachkräfte gegenüber Eltern ihr (vermeintliches) Fehlverhalten offenlegen. Hingegen wird von den Kindern kein Bezug darauf genommen, inwieweit Eltern das Verhalten der Kinder im familiären Kontext den Fachkräften gegenüber thematisieren. Das Elterngespräch wird daher als ausschließlich auf die Betreuungseinrichtung Bezug nehmend verstanden. Gleichzeitig wird den Eltern ein hohes Maß an Entscheidungsmacht darüber zugesprochen, inwieweit Kinder über die Inhalte der Elterngespräche informiert werden oder inwiefern sie teilnehmen dürfen resp. müssen. Die Fachkräfte werden in diesem Zusammenhang nicht als mögliche Ansprechpartner/innen verstanden, Informationen an die Kinder weiterzutragen. Somit lässt sich konstatieren, dass sowohl Eltern als auch Fachkräfte für generationale Asymmetrien im Rahmen von Elterngesprächen ausschlaggebend sind. Die Ergebnisse zeigen nicht nur eine hohe Anschlussfähigkeit zu Studien aus dem Primarbereich, die Perspektiven von Schülerinnen und Schülern in den Blick nehmen (u.a. Betz et al. 2019b; Stiller 2020), sondern auch zu solchen, die Gesprächsanalysen zwischen Lehrkräften, Eltern und Schülerinnen und Schülern zum Gegenstand haben (z. B. Bonanati 2018; Hauser und Mundwiler 2015; Mundwiler 2017).

Eingeschränkt wird der Erkenntnisgewinn durch die im Rahmen des explorativen Designs gewählte Samplegröße, so dass die vorliegende Analyse als Annäherung an den Forschungsgegenstand einzuordnen ist. Demzufolge können die Ergebnisse als Ausgangspunkt weiterer Forschungen gesehen werden, die Kinder im Verhältnis von Familie und Kindertageseinrichtung mitdenken. So könnten Studien zu den Perspektiven von Kindern auf die Kommunikation zwischen Eltern und Fachkräften beispielsweise einen stärker partizipatorischen Blick einnehmen und dabei auch weitere Formen der Zusammenarbeit von Familie und Kindertageseinrichtung berücksichtigen. Auch bedarf es Forschungen, die sich aus ungleichheitstheoretischer Perspektive Elterngesprächen als Gegenstand frühpädagogischer Praxis widmen. Fallstudien könnten unter anderem rekonstruieren, ob Kinder Elterngespräche entlang ihrer sozialen Herkunft unterschiedlich einschätzen, für sich zu nutzen wissen und inwieweit dies auf ungleiche Beteiligungsmöglichkeiten zurückzuführen ist.

Danksagung Die Autorin dankt den hilfreichen Rückmeldungen der Betreuenden sowie der wissenschaftlichen Peers zu einer früheren Version des Beitrags.

Funding Open Access funding enabled and organized by Projekt DEAL.

Open Access Dieser Artikel wird unter der Creative Commons Namensnennung 4.0 International Lizenz veröffentlicht, welche die Nutzung, Vervielfältigung, Bearbeitung, Verbreitung und Wiedergabe in jeglichem Medium und Format erlaubt, sofern Sie den/die ursprünglichen Autor(en) und die Quelle ordnungsgemäß nennen, einen Link zur Creative Commons Lizenz beifügen und angeben, ob Änderungen vorgenommen wurden.

Die in diesem Artikel enthaltenen Bilder und sonstiges Drittmaterial unterliegen ebenfalls der genannten Creative Commons Lizenz, sofern sich aus der Abbildungslegende nichts anderes ergibt. Sofern das betreffende Material nicht unter der genannten Creative Commons Lizenz steht und die betreffende Handlung nicht nach gesetzlichen Vorschriften erlaubt ist, ist für die oben aufgeführten Weiterverwendungen des Materials die Einwilligung des jeweiligen Rechteinhabers einzuholen. 
Weitere Details zur Lizenz entnehmen Sie bitte der Lizenzinformation auf http://creativecommons.org/ licenses/by/4.0/deed.de.

\section{Literatur}

Alanen, L. (2011). Explorations in generational analysis. In L. Alanen \& B. Mayall (Hrsg.), Conzeptualizing child-adult relations (S. 11-22). London, New York: Routledge/Falmer.

Alasuutari, M. (2014). Voicing the children? A case study in Finnish early childhood education. Childhood, 21(2), 242-259.

Andresen, S. (2012). Was und wie Kinder erzählen. Potential und Grenzen qualitativer Interviews. Frühe Bildung, 1(3), 137-142.

Betz, T. (2015). Das Ideal der Bildungs- und Erziehungspartnerschaft. Kritische Fragen an eine verstärkte Zusammenarbeit zwischen Kindertageseinrichtungen, Grundschulen und Familien. Gütersloh: Bertelsmann Stiftung.

Betz, T., Bischoff, S., Eunicke, N., Kayser, L. B., \& Zink, K. (2017). Partner auf Augenhöhe? Forschungsbefunde zur Zusammenarbeit von Familien, Kitas und Schulen mit Blick auf Bildungschancen. Gütersloh: Bertelsmann Stiftung.

Betz, T., Bischoff-Pabst, S., Eunicke, N., \& Menzel, B. (2019a). Kinder zwischen Chancen und Barrieren. Zusammenarbeit zwischen Kita und Familie: Perspektiven und Herausforderungen. Forschungsbericht 1. Gütersloh: Bertelsmann Stiftung.

Betz, T., Bischoff-Pabst, S., Eunicke, N., \& Menzel, B. (2019b). Kinder zwischen Chancen und Barrieren. Zum Verhältnis von Schule und Familie aus der Sicht von Kindern: ihre Perspektiven, ihre Positionen. Forschungsbericht 2. Gütersloh: Bertelsmann Stiftung.

Betz, T., Bollig, S., Cloos, P., Krähnert, I., \& Zehbe, K. (2021). Kinder, Eltern, pädagogische Fachkräfte: Programmatiken und Praktiken institutioneller Verhältnisverschiebungen zwischen Familie und Kindertageseinrichtungen. In Sektion Sozialpädagogik und Pädagogik der frühen Kindheit (Hrsg.), Familie im Kontext kindheits- und sozialpädagogischer Institutionen (S. 85-99). Weinheim, Basel: Beltz Juventa.

Bonanati, M. (2018). Lernentwicklungsgespräche und Partizipation. Rekonstruktionen zur Gesprächspraxis zwischen Lehrpersonen, Grundschülern und Eltern. Wiesbaden: Springer VS.

Brenneke, B., \& Tervooren, A. (2019). Gruppendiskussionen mit Kindern am Übergang vom Elementarzum Primarbereich. Methodologische Diskussionen und forschungspraktische Herausforderungen. In F. Hartnack (Hrsg.), Qualitative Forschung mit Kindern. Herausforderungen, Methoden und Konzepte (S. 193-237). Wiesbaden: Springer VS.

Brooker, L. (2007). Interviewing children. In M. G. Naughton, S. A. Rolfe \& I. Siraj-Blatchford (Hrsg.), Doing early childhood research. International perspectives on theory and practice (S. 162-178). Berkshire, New York: Open University Press.

Bühler-Niederberger, D. (2014). Sozialisation als generationales Ordnen - ein theoretischer und empirischer Versuch. Zeitschrift für Soziologie in der Erziehung und Sozialisation, 34(4), 339-354.

Bühler-Niederberger, D. (2020). Lebensphase Kindheit. Theoretische Ansätze, Akteure und Handlungsräume. Weinheim, Basel: Beltz Juventa.

Cameron, H. (2005). Asking the tough questions: a guide to ethical practices in interviewing young children. Early Child Development and Care, 175(6), 597-610.

Cloos, P., \& Karner, B. (2010). Erziehungspartnerschaft? Auf dem Weg zu einer veränderten Zusammenarbeit von Kindertageseinrichtungen und Familien. In ebd (Hrsg.), Erziehung und Bildung von Kindern als gemeinsames Projekt. Zum Verhältnis familialer Erziehung und öffentlicher Kinderbetreuung (S. 169-192). Baltmannsweiler: Schneider Verlag Hohengehren GmbH.

Cloos, P., Schulz, M., \& Thomas, S. (2013). Wirkung professioneller Bildungsbegleitung von Eltern. Rekonstruktive Forschungsperspektiven auf kindheitspädagogische Settings. In Kompetenzteam Wissenschaft des Bundesprogramms „Elternchance ist Kinderchance“, L. Correll \& J. Lepperhoff (Hrsg.), Frïhe Bildung in der Familie. Perspektiven der Familienbildung (S. 253-267). Weinheim, Basel: Beltz Juventa.

Croizer, G. (1999). Parental Involvment: who wants it? International Studies in Sociology of Education, 9(3), 219-238.

Ericsson, K., \& Larsen, G. (2002). Adults as resources and adults as burdens. The strategies of children in the age of school-home collaboration. In R. Edwards (Hrsg.), Children, home and school. Regulation, autonomy or connection? (S. 92-105). London, New York: Routledge/Falmer. 
Hauser, S., \& Mundwiler, V. (Hrsg.). (2015). Sprachliche Interaktionen in schulischen Elterngesprächen. Bern: Hep.

Heinzel, F. (2012a). Der Blick auf Kinder. In H. de Boer \& S. Reh (Hrsg.), Beobachtung in der Schule Beobachten lernen (S. 173-188). Wiesbaden: Springer VS.

Heinzel, F. (2012b). Gruppendiskussion und Kreisgespräche. In ebd (Hrsg.), Methoden der Kindheitsforschung. Ein Überblick über Forschungszugänge zur kindlichen Perspektive (S. 104-115). Weinheim, Basel: Beltz Juventa.

Heinzel, F., Kränz-Nagel, R., \& Mierendorff, J. (2012). Sozialwissenschaftliche Kindheitsforschung - Annäherung an einen komplexen Forschungsbereich. Zeitschrift für Religionspädagogik, 11(1), 9-37.

Honig, M.-S. (1999). Forschung „,vom Kinde aus“"? Perspektivität der Kindheitsforschung. In M.-S. Honig, A. Lange \& H. R. Leu (Hrsg.), Aus der Perspektive von Kindern? Zur Methodologie der Kindheitsforschung (S. 33-50). Weinheim, München: Juventa.

Honig, M.-S. (2018). Kindheit als praxeologisches Konzept. Von der generationalen Ordnung zu generationierenden Praktiken. In J. Budde, M. Bittner, A. Bossen \& G. Rißler (Hrsg.), Konturen praxistheoretischer Erziehungswissenschaft (S. 193-209). Weinheim, Basel: Beltz Juventa.

Karila, K. (2006). The significance of parent-practitioner interaction in early childhood education. Zeitschrift für qualitative Bildungs-, Beratungs- und Sozialforschung, 7(1), 7-24.

Kelle, H. (2018). Generationale Ordnung als Proprium von Erziehungswissenschaft und Kindheitssoziologie. In T. Betz, S. Bollig, M. Joos \& S. Neumann (Hrsg.), Institutionalisierung von Kindheit. Childhood Studies zwischen Soziologie und Erziehungswissenschaft (S. 38-52). Weinheim, Basel: Beltz Juventa.

Kesselhut, K. (2015). Machtvolle Monologe. ,Elterngespräche“ als Herstellungsort von Differenz. In P. Cloos, K. Koch \& C. Mähler (Hrsg.), Entwicklung und Förderung in der frühen Kindheit. Interdisziplinäre Perspektiven (S. 192-206). Weinheim, Basel: Beltz Juventa.

Kuckartz, U. (2018). Qualitative Inhaltsanalyse, Methoden, Praxis, Computerunterstützung. Weinheim, Basel: Beltz Juventa.

Kuhn, M., Machold, C., \& Schulz, M. (2018). Die Multifunktionalität von Bildungsdokumentationen. Zur Materialität von Elterngesprächen im elementarpädagogischen Feld. Zeitschrift für Grundschulforschung, 11(1), 7-21.

Lange, A., \& Mierendorff, J. (2009). Methoden der Kindheitsforschung. Überlegungen zur kindheitssoziologischen Perspektive. In M.-S. Honig (Hrsg.), Ordnungen der Kindheit. Problemstellungen und Perspektiven der Kindheitsforschung (S. 183-210). Weinheim, München: Juventus.

Menzel, B. (2021). Die Akteurschaft von Kindern in Tür- und Angelgesprächen. Ergebnisse einer ethnographischen Studie im frühpädagogischen Setting. Diskurs Kindheits- und Jugendforschung, 16(1), $95-111$.

Mey, G. (2013). „Aus der Perspektive der Kinder“: Ansprüche und Herausforderungen einer programmatischen Konzeption in der Kindheitsforschung. Psychologie und Gesellschaftskritik, 37(3/4), 53-71.

Mundwiler, V. (2017). Beurteilungsgespräche in der Schule: eine gesprächsanalytische Studie zur Interaktion zwischen Lehrpersonen, Eltern sowie Schülerinnen und Schülern. Tübingen: Narr Francke Attempto.

Nentwig-Gesemann, I. (2002). Gruppendiskussionen mit Kindern: die dokumentarische Interpretation von Spielpraxis. Zeitschrift für qualitative Bildungs-, Beratungs- und Sozialforschung, 3(1), 41-63.

Nentwig-Gesemann, I., \& Mackowiak, K. (2012). Schwerpunkt: Interviews mit Kindern - methodische Herausforderungen und Potenziale. Frühe Bildung, 1(3), 121-124.

Stiller, K. T. (2020). „Elternarbeit“ aus Kindersicht. Habitusbildung im Krisenerleben. Wiesbaden: Springer VS.

Urban, M., Cloos, P., Meser, K., Objartel, V., Richter, A., Schulz, M., Thoms, S., Velten, J., \& Werning, R. (2015). Prozessorientierte Verfahren in der Bildungsdokumentation in inklusiven Settings. Opladen, Berlin, Toronto: Barbara Budrich.

Weltzien, D. (2012). Gedanken im Dialog entwickeln und erklären: Die Methode dialoggestützter Interviews mit Kindern. Frühe Bildung, 1(3), 143-149.

Zehbe, K. (2021). Dealing with transition in formal meetings of parents and pedagogic staff. European Early Childhood Education Research Journal, 29(4), 569-581. 